\title{
¿Son los AgNOR's una herramienta útil como marcadores pronósticos de cáncer oral?
}

Leyva-Huerta ER*, Flores-Flores G**, Gaitán-Cepeda LA***

\section{RESUMEN}

Antecedentes. Es necesario contar con herramientas pronósticas para identificar pacientes oncológicos orales de alto riesgo de progresión a estadio terminal.

Objetivo. Establecer si el número, forma y tamaño de AgNOR's se relacionan con el grado de malignidad de carcinomas de células escamosas de cavidad oral (CCECO) y con el tiempo de sobrevida de pacientes oncológicos. Material y métodos. 19 casos de CCECO (Departamento de Patología, Instituto nacional de Cancerología, México), 3 de bajo grado, 7 de grado intermedio y 9 casos de alto grado de malignidad, fueron utilizados. De sus archivos médicos se obtuvieron lo siguientes datos: edad al momento del diagnóstico, género, localización del tumor primario, y tiempo de sobrevida. Del material biológico disponible se obtuvieron cortes seriados a $6 \mu \mathrm{m}$ que se tiñeron con la técnica de impregnación argéntica para Regiones de Organización Nucleolar de Ploton. De cada caso se determinó la cantidad, tamaño y forma de AgNOR's. Se formaron grupos de estudio en relación al tiempo de sobrevida: $\leq 11$ meses (8 casos); y $\geq 12$ meses de sobrevida (11 casos); y en relación al promedio de AgNOR's por núcleo en: $\leq 8$ AgNOR's (8 casos), y $\geq 9$ AgNOR's (12 casos). Se utilizaron las pruebas estadística de $x^{2}$, correlación de Pearson's, $t$ de student y regresión logística multivariada ( $<<0,05_{\text {IC95\%) }}$. Resultados. Promedio de AgNOr's por núcleo = 9,05 ( $\pm 2,01)$; promedio de tamaño $=3,5 \mathrm{~nm}$. El grupo con $\geq 9$ AgNOR's se relaciona con CCECO de alto grado de malignidad $(p<0,05)$ pero no con el tiempo de sobrevida. Se sugiere que la cantidad de AgNOR's puede ser útil como una herramienta auxiliar para identificar CCECO de alto grado de malignidad.

Palabras clave: Cáncer oral, AgNOR’s, Marcador pronóstico.

\section{SUMMARY}

Background. It has been observes an increase in oral cancer cases, therefore it is necessary to have prognostic tools to identify oral cancer patients with high risk to progress to terminal stage.

Aim. To establish if AgNOR's are related to to histological grade of squamous cells carcinoma of oral cavity (SCCOC) and with the survival time of patients suffering SCCOC.

Material and Methods. Nineteen archives cases of SCCOC, (Pathology Department, National Institute of cancer, México City), 3 cases of low grade, 7 cases of middle grade, and 9 cases of high grade of malignancy. From medical files it was obtained age at moment of diagnosis, gender, site of primary tumour and time of survival. From biological samples was obtained cut at $6 \mu \mathrm{m}$ and stained with argental impregnation technique to Nucleolar Organization Regions of Ploton. From each case was determinate amount, size, and shape of AgNOR's. The cases were grouped in regard to survival in: $\leq 11$ months group ( 8 cases); and $\geq 12$ months

\footnotetext{
* $\quad$ Subjefe de Investigación. División de Estudios de Postgrado e Investigación. Fac. Odontología. UNAM. ** Laboratorio de Patología, (Práctica Privada). Tuxtla Gutiérrez, México.

*** Jefe del Laboratorio de Patología Clínica y Experimental. División de Estudios de Postgrado e Investigación. Fac. Odontología, UNAM.
} 
group (11 cases), and in regard to AgNOR's average in: $\leq 8$ AgNOR's group (8 cases) and $\geq 9$ AgNOR's group (12 cases). The follows statistical tests were done: $\mathrm{x}^{2}$, Pearson's correlation, $t$ student and multivariate logistic regression $\left(\mathrm{p}<0.05_{\text {IC95\% }}\right)$.

Results. AgNOR's mean per nuclei $=9.05$ ( \pm 2.01$)$; AgNOR's mean size $=3.5 \mathrm{~nm}$. The $\geq 9$ AgNOR's group was related to high grade SCCOC $(p<0.05)$ but not with survival time. It is suggest that quantity of AgNOR's could be useful as an auxiliary tool to identify high grade SCCOC.

Key words: Oral cancer, AgNOR's, Prognostic markers.

Fecha de recepción: Octubre 2007.

Aceptado para publicación: Octubre 2007.

Leyva-Huerta ER, Flores-Flores G, Gaitán-Cepeda LA. ¿Son los AgNOR's una herramienta útil como marcadores pronósticos de cáncer oral? Av. Odontoestomatol 2008; 24 (3): 211-218.

\section{INTRODUCCIÓN}

En años recientes se ha observado en países desarrollados un incremento en la incidencia de casos de cáncer, especialmente entre individuos por debajo de 60 años. (1). En nuestro país, México, también se ha observado ese incremento. Se ha reportado un incremento el número de muertes directamente atribuibles a cáncer relacionado con asociado a tabaquismo crónico (2), en la frecuencia de cáncer de mama, (3), así como de cáncer de cérvix uterino (4). Estos dos últimos padecimientos constituyen la primera causa de muerte de mujeres en nuestro país.

En el caso del cáncer oral también existe evidencia que sugiere un incremento en la prevalencia, principalmente en sujetos por debajo de 40 años (5), así como en sujetos jóvenes sin antecedentes de exposición a factores de riesgo carcinogénicos conocidos (6). Por lo anterior se hace imperativo el contar con herramientas diagnósticas y pronósticas confiables, tanto para identificar sujetos de alto riesgo de padecer cáncer oral, así como para identificar pacientes oncológicos orales con mayor probabilidad de entrar a corto plazo a fase terminal y muerte.

Uno de los métodos más comúnmente utilizados para establecer el comportamiento de malignidad de neoplasias es la graduación histológica y su combinación con el sistema clínico Tumor, Nódulos linfático, Metástasis (TNM). Para establecer el grado de malignidad de las neoplasias son utilizados parámetros cualitativos soportados por aspectos citomorfológicos, por lo anterior este método tiene un fuerte componente subjetivo. El contar con una herramienta cuantitativa confiable que soporte establecer las observaciones subjetivas podría contribuir a establecer de manera confiable el pronóstico del paciente con cáncer oral.

Por otra parte, las regiones organizadoras nucleolares (RON's) son resultado de la concentración de rRNA (RNA 28S, 18S, 58S), de tal forma, la cantidad de RON's se incrementa en células donde la tasa de mitosis se encuentra incrementada (7-11). Las células neoplásicas tienen una tasa de mitosis incrementada, por lo tanto tendrán mayor cantidad de rRNA, y mayor cantidad de NOR. Uno de los más importantes parámetros histológicos para determinar la velocidad de crecimiento en una neoplasia es la tasa de mitosis, o índice mitótico. Tumores de alto grado de malignidad muestran altas tasas de mitosis. Una técnica establecida para cuantificar RON's es la técnica de regiones organizadoras nucleolares argirofílicas (AgNOR's por sus siglas en inglés) (12). Por lo que los AgNOR's pueden ser considerados como evidencia morfológica de un incremento en la división celular.

Por lo anterior el principal objetivo del presente trabajo es establecer si la cantidad de AgNOR's puede ser considerada como una herramienta pronostica 
útil en pacientes que padecen carcinomas de células escamosas de la cavidad oral (CCECO).

\section{MATERIAL Y MÉTODOS}

Diez y nueve casos de CCECO provenientes del servicio de Patología, Instituto Nacional de Cancerología, México fueron utilizados. Para ser incluidos, los casos deberían contener material biológico en suficiente cantidad incluido en parafina y cortes histológicos teñidos con técnica de tinción de hematoxilina-eosina (H-E). Del archivo médico de cada caso fueron obtenidos los siguientes datos: edad al momento del diagnóstico de CCECO, género, localización, estadio clínico de acuerdo a la clasificación TNM, evolución previo a su diagnóstico, tiempo de sobrevenida (tomado como el lapso de tiempo desde el diagnóstico hasta la muerte) y que la causa de muerte fuera directamente asociado a el CCECO. El tipo y tiempo de tratamiento no fue tomado en cuenta.

De cada una de las muestras se obtuvieron cortes adicionales a $6 \mu \mathrm{m}$ para ser teñidos con $\mathrm{H}$-E y con la técnica de impregnación argéntica para NOR's de Ploton (13). Brevemente, esta técnica consiste en colocar la muestra en gelatina al $2 \%$ en solución acuosa de ácido fórmico al $1 \%$ mezclado en dos partes de nitrato de plata al $59 \%$, a $30^{\circ} \mathrm{C}$ por 45 minutos en un cuarto obscuro.

Las muestras fueron revisadas bajo el microscopio de luz para ser corroborado el diagnóstico de CCECO por un experto patólogo oral (ERLH) y entonces clasificar al tumor de acuerdo a los criterio establecidos por Jacobson. Para el conteo de AgNOR's se escogieron aleatoriamente, de cada caso, 100 queratinocitos bajo un aumento de $1.000 \mathrm{X}$ y fotomicrografiados. En las imágenes obtenidas fueron contados la cantidad de AgNOR's por queratinocito, medido el diámetro y forma de AgNOR's, medido el tamaño nucleolar, utilizando para tal fin el programa computacional ImageScan (14). Como tejido control se utilizó hiperplasia linfoide linfocítica.

Para la identificación y amplificación de rRNA $18 \mathrm{~S}$ de cada una de las muestras, cuatro cortes de $50 \mu$ fueron obtenidas, para ser procesados con la técni- ca de la reacción en cadena de la polimerasa. Brevemente, posterior a la desparafinación con xileno, el RNA y DNA fueron purificados con K-proteinasa durante toda la noche. El RNA y DNA fueron purificados entonces con tiocianato de guanidina y precipitado con etanol. De tal forma se obtuvieron 5 nanogramas de RNA que fue utilizado para sintetizar DNA complementario por transcriptasa reversa. La secuencia de oligonucleótidos específicos para RNA ribosomal $18 \mathrm{~S}$ fue:

\section{5'ATG / CTC / TTA / GCT / GAG / TGT / CC3' \\ 5'AAC/ TAC / GAC / GGT / ATC / TGAQ / TC3'}

Una secuencia de deshidrogenasa de gliceraldehido3-fosfato (GAPDH) fue usado como control interno de amplificación. El producto de la amplificación para $18 \mathrm{~S}$ fue de 344 pb y para GAPDH, 110 pb. Los productos de la amplificación fueron separados con geles de acrilamida a los $4 \%$ teñidos con etil brómide $y$, entonces, fotografiados.

Para establecer la posible correlación entre el número, tamaño y forma de AgNOR's, y amplificación de rRNA $18 \mathrm{~S}$ con el tiempo de sobrevida y grado de malignidad se utilizó la prueba de correlación de Pearson. Para la posible asociación entre las variables no-paramétricas la prueba de $\mathrm{x}^{2}$ fue utilizada $\left(p<0,05_{\mathrm{IC95}}\right)$. Para la comparación estadística entre variables paramétricas un prueba $t$ de student fue utilizada con un nivel de confianza del 95\%. Para establecer una posible asociación de tiempo de sobrevida con las diferentes variables, un aprueba de regresión logística multivariada $\left(p<0,05{ }_{\text {IC95\% }}\right)$ fue realizada. El protocolo de investigación fue aprobado por el comité de Bioética de las instituciones participantes.

\section{RESULTADOS}

De los 19 casos de CCECO que cumplieron los criterios de inclusión; 4 provenían de mujeres (promedio de edad al momento del diagnóstico 66.5 años; rango de 45-86 años) y 15 de varones (promedio de edad al momento del diagnóstico 63 años; rango de 42-76 años). Con respecto al estadio clínico (TNM) al momento del diagnóstico, el estadio mas frecuente fue T3NOMO con 4 casos, 8 casos $(42,1 \%)$ fueron 
estadio T4; 10 casos $(52,6 \%)$ no mostraron linfonodos positivos (N0) y únicamente un caso mostró metástasis. Topográficamente ocho casos correspondieron a carcinomas de células escamosas de lengua; 7 se ubicaron en el piso de boca, 3 en mucosa bucal y un caso se ubicó en la encía.

El promedio del tiempo de sobrevida, independientemente del tipo de tratamiento, fue de 14,6 meses (desviación estándar $\pm 10,6$; rango 2 - 48 meses). Con fines de investigación los casos se agruparon respecto a la sobrevida como sigue: grupo con $\leq 11$ meses de sobrevida, formado por 8 casos; y grupo con $\geq 12$ meses de sobrevida formado por 11 casos. En relación a su grado histológico, 3 (15,8\%) correspondieron a tumores de bajo grado de malignidad, 7 $(36,8 \%)$ fueron diagnosticados como carcinomas de células escamosas de grado intermedio de maligni- dad y $9(47,4 \%)$ correspondieron a tumores de alto grado de malignidad.

El número total de queratinocitos analizados fue de 1.900. El promedio de AgNOR's por nucleolo fue de 9,05 (desviación estándar $\pm 2,01$, rango de 6-12). El diámetro promedio de los AgNOR's fue de 3,5 nm (rango 2-18 nm).

La amplificación de rRNA $18 \mathrm{~S}$ mostró que en tres casos no hubo amplificación, 4 casos mostraron amplificación leve, 11 casos mostraron amplificación y 1 caso mostró sobreexpresión. El total de datos de los casos analizados se muestra en la tabla 1.

Con propósito de investigación y de acuerdo al principal objetivo del presente reporte, los casos fueron agrupados de acuerdo al número de AgNOR's por

\begin{tabular}{|c|c|c|c|c|c|c|c|c|c|c|}
\hline & CARA & $\begin{array}{l}\text { ГABLA } \\
\text { 'TERÍST } \\
\text { ARCINO }\end{array}$ & $\begin{array}{l}\text { - LOCAL } \\
\text { ICAS DE } \\
\text { MA DE C }\end{array}$ & $\begin{array}{l}\text { IZACIÓ } \\
\text { AGNOF } \\
\text { ÉLULAs }\end{array}$ & $\begin{array}{l}\text { Y, ESTADIC } \\
\text { S Y DATOS } \\
\text { ESCAMOS }\end{array}$ & $\begin{array}{l}\text { CLÍNICO, T } \\
\text { DEMOGRÁ } \\
\text { AS DE CAVI }\end{array}$ & $\begin{array}{l}\text { MPO DE } \\
\text { COS DE } \\
\text { AD ORAL }\end{array}$ & $\begin{array}{l}\text { OBREVIL } \\
\text { OS } 19 \mathrm{CA} \\
\text { ANALIZAL }\end{array}$ & $\begin{array}{l}\text { A, } \\
\text { SOS D } \\
\text { OS }\end{array}$ & \\
\hline Caso & $\begin{array}{c}\text { Edad } \\
\text { (a) }\end{array}$ & Género & $\begin{array}{l}\text { Estadio } \\
\text { clínico }\end{array}$ & $\begin{array}{l}\text { Locali- } \\
\text { zación }\end{array}$ & $\begin{array}{c}\text { Tiempo de } \\
\text { sobrevida } \\
\text { (m) }\end{array}$ & $\begin{array}{l}\text { Grado de } \\
\text { malignidad }\end{array}$ & $\begin{array}{l}\text { AgNOr's } \\
\text { (por } \\
\text { núcleo) }\end{array}$ & $\begin{array}{c}\text { Tamaño } \\
\text { AgNOr's } \\
(\mathrm{nm})\end{array}$ & $\begin{array}{c}\text { rRNA } \\
18 S\end{array}$ & GAPDH \\
\hline 1 & 75 & V & T4N1M0 & $\mathrm{L}$ & 18 & Intermedio & 6 & 1,92 & + & ++ \\
\hline 2 & 65 & V & T3NOMO & $\mathrm{L}$ & 13 & Intermedio & 6 & 1,92 & ++ & + \\
\hline 3 & 45 & $\mathrm{~F}$ & T3N1M0 & $\mathrm{L}$ & 5 & Alto & 7 & 1,88 & + & + \\
\hline 4 & 61 & V & TЗNOMO & L & 20 & Bajo & 6 & 1,92 & ++ & ++ \\
\hline 5 & 42 & V & T3NOMO & $\mathrm{L}$ & 20 & Alto & 8 & 1,92 & ++ & ++ \\
\hline 6 & 69 & $\mathrm{~F}$ & T4NOMO & SB & 14 & Intermedio & 9 & 1,70 & ++ & ++ \\
\hline 7 & 86 & $\mathrm{~F}$ & T2NOMO & MB & 48 & Intermedio & 9 & 1,92 & - & ++ \\
\hline 8 & 67 & V & T4N3M0 & $\mathrm{L}$ & 32 & Bajo & 9 & 1,88 & -+ & -+ \\
\hline 9 & 70 & V & T3N2M1 & SB & 11 & Bajo & 8 & 1,92 & ++++ & ++ \\
\hline 10 & 40 & V & T1NOMO & $\mathrm{L}$ & 8 & Bajo & 9 & 1,70 & -- & + \\
\hline 11 & 66 & V & Т3N2M0 & SB & 8 & Intermedio & 9 & 1,88 & ++ & ++ \\
\hline 12 & 63 & V & T4N1M0 & SB & 2 & Alto & 8 & 1,92 & ++ & ++ \\
\hline 13 & 64 & V & T3N2MO & SB & 10 & Bajo & 9 & 1,76 & + & ++ \\
\hline 14 & 86 & $\mathrm{~F}$ & $\mathrm{~T} 2 \mathrm{~N} 1 \mathrm{M} 0$ & $\mathrm{E}$ & 5 & Intermedio & 11 & 1,50 & + & + \\
\hline 15 & 76 & V & T4N1M0 & SB & 13 & Intermedio & 12 & 1,72 & ++ & ++ \\
\hline 16 & 73 & V & T4N2M0 & SB & 18 & Bajo & 11 & 1,72 & ++ & ++ \\
\hline 17 & 56 & $\mathrm{~F}$ & T2NOMO & $M B$ & 16 & Bajo & 11 & 1,72 & ++ & ++ \\
\hline 18 & 49 & V & T1N0M0 & $\mathrm{L}$ & 6 & Bajo & 12 & 1,72 & ++ & + \\
\hline 19 & 56 & V & TЗNOMO & MB & 12 & Bajo & 12 & 1,72 & ++ & ++ \\
\hline
\end{tabular}


queratinocito. De tal forma 2 grupos fueron formados: Grupo $\geq 9$ AgNOR's, compuestos por 12 casos; y Grupo $\leq 8$ AgNOR's, formados por 8 casos. Al relacionar el número de AgNOR's con el grado de malignidad se encontró que el 58,3\% de los casos del grupo $\geq 9$ AgNOR's correspondieron a casos de alto grado de malignidad. Por otra parte, únicamente el $28,5 \%$ de los casos con $\leq 8$ AgNOR's correspondieron a CCECO clasificados como de alto grado de malignidad $(p<0,05)$.

Cuando la cantidad de AgNOR's por núcleo se relaciona con el tiempo de sobrevida, no pudimos observar diferencias. Los resultados en ambos grupos fueron muy similares: $58,3 \%$ de los casos con $\geq 9$ AgNOR's tuvieron una sobrevida $=12$ meses; mientras que el $57,1 \%$ de casos con $=8$ AgNOR's tuvo un tiempo de sobrevenida $\geq 12$ meses. El grupo $\leq 8$ AgNOR's mostró un promedio de diámetro de AgNOR de 1.914 nm (desviación estándar $\pm 1,5 \%$ ) mientras que el grupo $\geq 9$ AgNOR's tuvo un promedio de diámetro de AgNOR de 1.745 nm (desviación estándar $\pm 1,1 \%)$. Esta diferencia fue estadísticamente significativa (p 0,001). La distribución de los grupos de estudio en función del grado de malignidad, tiempo de sobrevida y expresión de se muestran en la tabla 2.

En relación a la expresión de rRNA18S, 58,3\% de los casos con $\geq 9$ AgNOR's tuvieron expresión de rRNA18S, mientras que el $71,2 \%$ de los casos con $\leq 8$ AgNOR's tuvieron expresión de rRNA18S $(p>0,05)$.

\section{DISCUSIÓN}

El presente trabajo sugiere una posible asociación entre el número de AgNOR's y CCEOC de alto grado de malignidad y propone que la cantidad de AgNOR's puede ser considerada como una herramienta cuantitativa que contribuiría a la identificación de carcinomas orales de alto grado de malignidad.

Se ha propuesto a la presencia de NOR's como un marcador de proliferación celular $(8,10,11,15)$. La cantidad de AgNOR's depende de la cantidad de NOR's, del nivel de actividad trascripcional de RNA y del estadio del ciclo celular. A mediados de los 80's fue identificado genes-NOR en células neoplásicas y por lo tanto la posibilidad de que estos genes puedan ser amplificados (16). Se estableció que la amplificación de genes-NOR son proporcionales a el grado de malignidad de células neoplásicas, y que el tamaño de NOR's están relacionados al grado del nivel de trascripción (16). Por lo tanto, la transcripción del gen-rRNA puede ser propuesto como un importante marcador del tiempo de replicación de un tumor.

La utilidad de los AgNOR's en relación al grado de malignidad ha sido propuesto en dos maneras: 1) como marcador de malignidad y 2) como marcador pronóstico de tumores malignos. En el caso de la primera situación AgNOR's han sido utilizados como marcadores de proliferación queratinocítica (12), y contribuyendo a la identificación temprana de leucoplasias orales potencialmente malignas (18-20).

\section{TABLA 2.- ASOCIACIÓN DE CANTIDAD DE AGNOR'S CON GRADO DE MALIGNIDAD, TIEMPO DE SOBREVIDA Y EXPRESIÓN DE RNA $18 S$.}

\begin{tabular}{|c|c|c|c|c|c|c|c|c|}
\hline \multirow{2}{*}{ Grupo } & \multicolumn{3}{|c|}{ Grado de malignidad } & \multicolumn{2}{|c|}{ Sobrevida (meses) } & \multicolumn{3}{|c|}{ RNA18S } \\
\hline & Alto & Interm. & Bajo & $\leq 11$ & $\geq 12$ & - & + & ++ \\
\hline $\begin{array}{l}\geq 9 \text { AgNORs } \\
(n=12)\end{array}$ & $\begin{array}{c}7 \\
58,3 \%\end{array}$ & $\begin{array}{c}5 \\
41,6 \%\end{array}$ & 0 & $\begin{array}{c}5 \\
41,6 \%\end{array}$ & $\begin{array}{c}7 \\
58,3 \%\end{array}$ & $\begin{array}{c}3 \\
25 \%\end{array}$ & $\begin{array}{c}2 \\
16,6 \%\end{array}$ & $\begin{array}{c}7 \\
58,3 \%\end{array}$ \\
\hline \multirow[t]{2}{*}{$\begin{array}{l}\leq 8 \text { AgNORs } \\
(n=7)\end{array}$} & $\begin{array}{c}2 \\
28,6 \%\end{array}$ & $\begin{array}{c}2 \\
28,6 \%\end{array}$ & $\begin{array}{c}3 \\
42,9 \%\end{array}$ & $\begin{array}{c}3 \\
42,9 \%\end{array}$ & $\begin{array}{c}4 \\
57,1\end{array}$ & 0 & $\begin{array}{c}2 \\
28,5 \%\end{array}$ & $\begin{array}{c}5 \\
71,4 \%\end{array}$ \\
\hline & p 0,003 & & & & & & & \\
\hline
\end{tabular}


Sin embargo la literatura científica muestra datos controversiales acerca de la cantidad de AgNOR's que deben de mostrar las células epiteliales para ser consideradas como potencialmente malignas o inclusive como carcinomas. Con respecto a el cáncer de cavidad oral, ha sido propuesto que 2,37 es la cantidad de AgNOR's para distinguir un epitelio displásico de un epitelio no-displásico (12). Por otra parte un número mayor a 4 AgNOR's por núcleo ha sido sugerido para diferenciar epitelio normal de epitelio displásico; y entre epitelio normal de CCECO (21). Nuestros resultados muestran un promedio de 9,5 AgNOR's por núcleo, similar a lo previamente reportado $(8,37)$ para CCECO $(22)$.

Se conoce que tanto el grado de malignidad de un tumor, como el estadio clínico de los pacientes oncológicos, son predoctores de supervivencia así como del tiempo libre de tumor (23). Por lo tanto la utilidad de los AgNOR's como marcadores pronósticos de evolución de CCECO deben de estar soportadas por una estrecha asociación entre estadio histológico/clínico con AgNOR's. La literatura científica muestra datos contradictorios al respecto. Por una parte no se ha reportado correlación entre cantidad de AgNOR's y estadio clínico (23), mientras que por otra parte se ha reportado que tumores malignos en estadio clínico T3/T4 están asociados a un número igual o mayor a 2,8 AgNOR's (19). Ninguno de los casos revisados por nosotros tuvieron un promedio de AgNOR's por núcleo menor a 4 a pesar de que ninguno de los casos se encontraba en el momento de la biopsia en estadio T3/T4. Esta aparente disparidad pudiera estar asociada a diferencias metodológicas aunque pudiera estar asociado también a diferentes comportamientos etnorraciales, como ha sido sugerido recientemente (24). Sin embargo el análisis de nuestros datos muestran una relación estadísticamente significativa $(p<0,05)$ entre una cantidad $\geq 9$ AgNORs's y carcinomas de alto grado de malignidad. Adicionalmente encontramos que carcinomas de alto grado de malignidad muestran un tamaño menor de AgNOR's que los encontrados en CCECO de bajo grado de malignidad. Se ha sugerido que epitelios severamente displásicos muestran mayores cantidades de AgNOR's así como AgNOR's pequeños que los observadores en células epiteliales normales $(6,9)$. Sin embargo esta última aseveración es controversial, ya que recientemente se ha propuesto que el tamaño de los AgNOR's es mayor en tumores de alto grado de malignidad (25).

Respecto a su valor como marcador pronóstico, la literatura científica también muestra datos controversiales (26). Ha sido reportado que pacientes con CCECO con tiempo de sobrevida menor a los 2 años muestran un promedio de AgNOR's por núcleo de 7,1, mientras por otro lado pacientes con mejor pronóstico muestran un promedio de AgNOR's por núcleo de 6.14 (27). Ha sido demostrado que el coeficiente de variación de número y área de AgNOR's están fuertemente relacionados al curso clínico de CCECO (28). Nosotros no fuimos capaces de encontrar asociación entre número, tamaño y diámetro de AgNOR's y tiempo de sobrevida.

Un factor que pudiera influenciar nuestros resultados es el tipo y tiempo bajo tratamiento. Es conocido que el tipo de tratamiento, especialmente la combinación de quimioterapia y radioterapia esta asociado a un incremento en la sobrevida de pacientes con cáncer oral y orofaríngeo (29). Otra explicación putativa para justificar esta aparente discrepancia pudiera ser que los grupos de estudios se traslapan. Esto es, que no exista un valor práctico de AgNOR's para ser aplicados en lesiones individuales tal y como fue propuesto por Warnakulasuriya (22). Además, parece ser que no todas las características morfológicas de los AgNOr's son útiles para identificar lesiones con diferentes grados de displasia, incluyendo carcinomas microinvasores (30).

Nuestros resultados sugieren que la cantidad de AgNOR's puede ser útiles como auxiliares en la identificación de CCECO de alto grado de malignidad y de esta forma contribuir a soportar decisiones terapéuticas. Además sugieren que un número $\geq 9$ AgNOR's por núcleo pudiera ser un punto para clasificar carcinomas orales agresivos cuyas características histopatológicas no permitan, sin lugar a dudas, establecer su grado de malignidad. El análisis de AgNOR's pudiera ser una herramienta complementaria útil para clasificar casos complejos de cáncer oral (2).

El presente reporte sugiere que la cantidad de AgNOR's esta directamente relacionada con CCECO de alto grado de malignidad. Nuestro país, México, 
es un país en vías de desarrollo que necesita optimizar los recursos destinados a las terapias altamente especializadas de pacientes con cáncer. Por lo anterior, las instituciones de salud están reforzando tanto las estrategias de prevención de cáncer como los programas de tratamiento (31). Establecer un pronóstico, soportado por evidencia confiable, pudiera contribuir en una importante manera a optimizar los recursos de las instituciones de salud.

En conclusión el presente trabajo muestra que la cantidad de AgNOR's puede ser considerado como una herramienta útil para establecer el grado de malignidad de CCECO.

\section{BIBLIOGRAFÍA}

1. Gillison ML: Current topics in the epidemiology of oral cavity and oropharyngeal cancers. Head Neck 2007;29:779-92.

2. Tovar-Guzman VJ, Barquera S, Lopez-Antunano FJ: Mortality trends in cancer attributable to tobacco in Mexico. Salud Publ Mex 2002;44 Suppl 1:S20-8.

3. Rodriguez Cuevas SA, Capurso Garcia M: Epidemiology of breast cancer. Ginecol Obstet Mex 2006; 4:585-93.

4. Pina-Sanchez P, Hernandez-Hernandez DM, Lopez-Romero R, et al: Human papillomavirusspecific viral types are common in Mexican women affected by cervical lesions. Int J Gynecol Cáncer. 2006;16:1041-7

5. Bell RB, Kademani D, Homer L, Dierks EJ, Potter BE: Tongue cancer: Is there a difference in survival compared with other subsites in the oral cavity? J Oral Maxillofac Surg 2007;65:229-36.

6. Chitapanarux I, Lorvidhaya V, Sittitrai P, et al: Oral cavity cancers at a young age: analysis of patient, tumor and treatment characteristics in Chiang Mai University Hospital.Oral Oncol 2006;42:83-8.

7. Piffkò J, Bànkfalvi A, Öfner D, Rasch D, Joos U, Schmid KW: Standardized AgNOR analysis of the invasive tumour front in oral squamous cell carcinomas. J Pathol 1997;182:450-6.

8. Derenzini $M$, Trerè D, Pession A, Govoni M, Sirri V, Chieco P: Nucleolar size indicates the rapidity of cell proliferation in cancer tissues. J Pathol 2000;191:181-6.

9. Crossen PE, Godwin JM: Rearrangement and possible amplification of the ribosomal RNA gene sites in the human chronic myelogenous leukemia cell line K562. Cancer Genet Cytogenet 1985;18:27-30.

10. Remmerbach TW, Weidenbach H, Muller C, et al: Diagnostic value of nucleolar organizer regions (AgNORs) in brush biopsies of suspicious lesions of the oral cavity. Anal Cell Pathol 2003;25:139-46.

11. Sano K, Takahashi H, Fujita S, et al: Prognostic implication of silver-binding nucleolar organizer regions (AgNORs) in oral squamous cell carcinoma. J Oral Pathol Med 1991;20:53-6.

12. Chimeno Zoth SA, Collet AM, Heber E, Schwint $\mathrm{AE}$, Itoiz ME: Early detection of alterations associated to oral cancer. Acta Odontol Latinoam 2000; 13:100-12.

13. Ploton D, Bendayan M, Adnet JJ: Ultrastructural localization of Ag-NOR proteins and nucleic acids in reticulated nucleoli. Biol Cell 1983;49:29-34.

14. Cabrini RL, Schwint AE, Mendez A, Femopase F, Lanfranchi $\mathrm{H}$, Itoiz ME: Morphometric study of nucleolar organizer regions in human oral normal mucosa, papilloma and squamous cell carcinoma. J Oral Pathol Med 1992;21:275-9.

15. Xie X, Clausen OP, Sudbo J, Boysen M: Diagnostic and prognostic value of nucleolar organizer regions in normal epithelium, dysplasia, and squamous cell carcinoma of the oral cavity. Cancer 1997;79:2200-8.

16. Warnakulasuriya KA, Johnson NW: Nucleolar organiser region (NOR) distribution as a diagnostic marker in oral keratosis, dysplasia and squamous cell carcinoma. J Oral Pathol Med 1993;22:77-81. 
17. Pich A, Chiarle R, Chiusa L, et al: Long-term survival of thymoma patients by histologic pattern and proliferative activity. Am J Surg Pathol 1995; 19:918-26.

18. Chattopadhyay A, Chawda JG, Doshi JJ. Silverbinding nucleolar organizing regions: a study of oral leukoplakia and squamous cell carcinoma. Int J Oral Maxillofac Surg 1994;23:374-7.

19. Pillai KR, Sujathan K, Madhavan J, Abraham EK: Significance of silver-stained nucleolar organizer regions in early diagnosis and prognosis of oral squamous cell carcinoma: a multivariate analysis. In Vivo 2005;19:807-12.

20. Shiboski CH, Shiboski SC, Silverman S Jr: Trends in oral cancer rates in the United States, 19731996. Community Dent Oral Epidemiol 2000; 28 : 249-56.

21. Piloni MJ, Keszler A, Itoiz ME: Agnor as a marker of malignant transformation in odontogenic keratocysts. Acta Odontol Latinoam. 2005;18:3742.

22. Kahn MA, Mincer HH, Dockter ME, HermannPetrin JM. Comparing flow cytometric analysis and nucleolar organizer region enumeration in archival oral premalignant lesions. J Oral Pathol Med 1993;22:257-62.

23. Wutzl A, Ploder O, Kermer C, Millesi W, Ewers R, Klug C: Mortality and causes of death after multimodality treatment for advanced oral and oropharyngeal cancer. J Oral Maxillofac Surg 2007;65:255-60.

24. Cruz GD, Salazar CR, Morse DE: Oral and pharyngeal cancer incidence and mortality among Hispanics, 1996-2002: the need for ethnoregional studies in cancer research. Am J Public Health 2006;96:2194-200.

25. Schwint AE, Gomez E, Itoiz ME, Cabrini RL. Nucleolar organizer regions as markers of incipient cellular alterations in squamous epithelium. $\mathrm{J}$ Dent Res 1993; 72: 1233-6.

26. Lo Muzio L, Mignogna MD, Staibano S, et al: Morphometric study of nucleolar organiser regions (AgNOR) in HPV-associated precancerous lesions and microinvasive carcinoma of the oral cavity. Oral Oncol 1997; 33: 247-59.

27. da Silva Fonseca LM, do Carmo MA: Identification of the AgNORs, PCNA and ck16 proteins in oral lichen planus lesions. Oral Dis 2001;7:344-8.

28. Chattopadhyay A, Ray JG, Caplan DJ: AgNOR count as objective marker for dysplastic features in oral leukoplakia. J Oral Pathol Med 2002;31: 512-7.

29. Bukhari $M H$, Niazi S, Khan SA, et al: Modified method of AgNOR staining for tissue and interpretation in histopathology. Int $\mathrm{J}$ Exp Path 2007;88:47-53.

30. Politi EN, Lazaris AC, Alexopoulou D, Lambropoulou S, Kavantzas N, Koutselini H: Morphometric analysis of AgNORs in thin-layer, liquid-based liver specimens. Anal Quant Cytol Histol 2004;26:187-93.

31. División Técnica de Información Estadística en Salud: IMSS in numbers. Cancers in insured population, 1990-2003. Rev Med Inst Mex Seguro Soc 2005;43:349-56.

\section{CORRESPONDENCIA}

Dra. Elba Rosa Leyva Huerta.

Laboratorio de Patología Clínica y Experimental.

División de Estudios de Postgrado e Investigación.

Fac. Odontología, UNAM.

Circuito Institutos s/n, Ciudad Universitaria.

Coyoacán, 04510, D.F.

México.

Tel/Fax (52) 56-22-5559

e-mail: docelbaleyva@hotmail.com 